\title{
Two Different Multi-Kernels for Fuzzy C-means Algorithm for Medical Image Segmentation
}

\author{
Nookala Venu ${ }^{\# 1}$, B. Anuradha ${ }^{* 2}$ \\ ${ }^{\# 1}$ Research Scholar, ${ }^{* 2}$ Professor, Department of Electronics and Communication Engineering, \\ Sri Venkateswara University, Tirupati-517502, India
}

\begin{abstract}
A new image segmentation using multi-hyperbolic and multi-Gaussian kernel based fuzzy c-means algorithm (MHMGFCM) is proposed for medical magnetic resonance image (MRI) segmentation. The integration of two hyperbolic tangent kernels and two Gaussian kernels are used in the proposed algorithm for clustering of images. The performance of the proposed algorithm is tested on OASIS-MRI image dataset. The performance is tested in terms of score, number of iterations (NI) and execution time (TM) under different Gaussian noises on OASIS-MRI dataset. The results after investigation, the proposed method shows a significant improvement as compared to other existing methods in terms of score, NI and TM under different Gaussian noises on OASIS-MRI dataset.
\end{abstract}

Keywords - FCM, multi-hyperbolic tangent function, Segmentation, multi-Gaussian Kernal, fuzzy, multiple-kernal.

\section{INTRODUCTION}

In image processing and computer vision, medical image segmentation is an active research area [1]. The process of clustering the image into non- overlaped, consistent regions is called the image segmentation. These regions are identical with respect to some features like texture, color, shape, intensity etc. Based on the features, the process of segmentation is separated into four groups: clustering (intensity), thresholding (intensity), region extraction (color or texture) and edge detection (texture).

In literature, several techniques are available for medical image segmentation. The previously available literature on segmentation methods are: thresholding techniques [2], clustering techniques [3], classifiers based techniques [4], region growing techniques [5], Artificial Neural Networks (ANNs) based techniques [6], Markov Random Field (MRF) [7] models atlas-guided techniques etc. Amongst the above discussed methods, the clustering based techniques show an importance in medical imaging research.

Clustering is a procedure for classifying patterns or objects in such a manner that samples of the same cluster are more comparable to one another than samples belonging to other clusters. There are two main clustering approachs: the hard clustering technique and the fuzzy clustering technique. MacQueen [8] has proposed the k-means clustering algorithm. The k-means is one of the hard clustering technique. The usual hard clustering techniues classify every point of the records set just to one cluster. As a effect, the results are often very crusty, i.e., in image clustering every pixel of the image goes to one cluster. However, in many real conditions, issues such as restricted spatial resolution, reduced contrast, partly cover intensities, noise and intensity in homogeneities decrease the efficiency of hard (crusty) clustering techniques. Fuzzy set theory [9] has bring in the idea of incomplete membership, explained by a membership function. Fuzzy clustering, as a soft segmentation technique, has been extensively analized and effectively applied in image segmentation and clustering [10]-[19]. Among the fuzzy clustering techniques, fuzzy cmeans (FCM) algorithm [10] is the generally well-liked technique which is used in image segmentation due to its robust features for uncertainty and can keep much more information as compared to hard segmentation techniques [11]. While the standared FCM algorithm works fit on most noise-free images, it is very aware to noise and other imaging artifacts, because it does not consider any data about spatial background.

Tolias and Panas [12] have proposed a fuzzy rule-based technique also known the ruled-based neighborhood improvement system to impress spatial constraints by post processing the FCM clustering results. Noordam et al. [13] have proposed a geometrically guided FCM (GG-FCM) algorithm which is a semi-supervised FCM technique. Here, a geometrical condition is used the local neighborhood of every pixel. Pham [14] has customized the FCM objective function by counting spatial punishment on the membership functions. The punishment term leads to an iterative algorithm, which is extremely comparable to the original FCM and allows the evaluation of spatially flat membership functions. Ahmed et al. [15] have proposed the FCM_S where the objective function of the standard FCM is modified in order to recompense the intensity in uniformity and permit the labeling of a pixel to be effected by the labels in its neighborhood. The disadvantage of FCM_S is that the neighborhood labeling is computed in every iteration step which is very time-consuming.

Chen and Zhang [16] have proposed FCM_S1 and FCM_S2 which are the two variants of FCM_S algorithm in order to decrease the computational complexity. These two techniques introduced the additional mean and medianfiltered image, respectively, which can be calculated in advance, to swap the neighborhood term of FCM_S. Thus, the implementation times of both FCM_S1 and FCM_S2 are significantly decresed. Further, they have enhanced the FCM_S objective function to more likely disclose intrinsic non-Euclidean structures in data and more robustness to noise. They then replaced the Euclidean distance by a kernel-induced distance and proposed kernel versions of FCM with spatial constraints also known as KFCM_S1 and KFCM_S2 [16]. However, the major disadvantage of 
FCM_S and its variants is that their parameters greatly change the finishing clustering results.

Szilagyi et al. [17] have proposed the enhanced FCM (EnFCM) algorithm to go faster the image segmentation procedure. The structure of the EnFCM is changed from that of FCM_S and its variants. First, a linearly-weighted sum image is created from both original image and every pixel's local neighborhood average gray scales. Then clustering is conducted on the basis of the gray scale histogram as an alternative of pixels of the summed image. Because the number of gray scales in an image is usually much less important than the number of its pixels, the computational complexisty of EnFCM algorithm is decresed, as the quality of the segmented image is equivalent to that of FCM_S [17]. Cai et al. [20] have proposed the fast generalized FCM algorithm (FGFCM) which uses the spatial information also known as the intensity of the local pixel neighborhood and the number of gray scales in an image. This technique forms a nonlinearly-weighted sum image from both original image and its local spatial and gray scale neighborhood. The computational complexity of FGFCM is very little, because clustering is carryed out on the basis of the gray scale histogram. The excellence of the segmented image is fine enhanced [20]. Yang and Tsai [21] have proposed the Gaussian kernel based FCM (GKFCM) for medical image segmentation. The proposed GKFCM algorithm becomes a generalized type of FCM, KFCM_S1 and KFCM_S2 algorithms and presents with more efficiency and robustness. Chen et al. [22] have proposed the multiple-kernel fuzzy Cmeans (FCM) (MKFCM) for image-segmentation problems. They have used the linear combination of multiple kernels as composite kernel.

Kannan et al. [23] have proposed the hyper tangent FCM (HTFCM)based image segmentation for breast images. They have used the hyper tangent function as objective function in place of original Euclidean distance on feature space. Venu et al. [24] have proposed the segmentation algorithm (HGFCM) which integrates the hyperbolic tangent and Gaussian kernel functions for MRI image segmentation.

The organization of the paper is given as follows. Sections I, presents the literature and related work of proposed clustering algorithms. The various methods which are available for cluster based segmentations are given in section II, Section III, presents the evaluation measures and dataset used in this paper. The experimental results and discussions are given in section IV. Conclusions are derived in section V.

\section{METHODS}

\section{A. Fuzzy C-means Algorithm}

Fuzzy c-means clustering technique is a simplification of the hard c-means algorithm yields enormously superior results in an image region clustering and object categorization. As in hard k-means algorithm, Fuzzy cmeans algorithm is based on the minimization of a standard function.
Let a matrix of $n$ data elements (image pixels), each of size $s(s=1)$ is represented as $X=\left(x_{1}, x_{2}, \ldots . ., x_{n}\right)$. FCM generates the clustering by iteratively minimizing the objective function given in Eq. (1).

Objective function: $O_{m}(U, C)=\sum_{i=1}^{c} \sum_{j=1}^{n} U_{i j}^{m} D^{2}\left(x_{j}, C_{i}\right)$

Constraint: $\sum_{i=1}^{c} U_{i j}=1 ; \quad \forall j$

Where, $U_{i j}$ is membership of the $j^{\text {th }}$ data in the $i^{\text {th }}$ cluster $C_{i}, m$ is fuzziness of the system $(m=2)$ and $D$ is the distance between the cluster center and pixel.

FCM algorithm

The algorithm for the FCM based clustering is given bellow.

Input: Raw image; Output: Segmented image;

- Randomly initialize the ( $c=3$ clusters) cluster centers $C_{i}$.

- The distance $D$ between the cluster center and pixel is calculated by using Eq. (3).

$$
D^{2}\left(x_{j}, C_{i}\right)=\left\|x_{j}-C_{i}\right\|^{2}
$$

- The membership values are calculated by using Eq. (4).

$$
U_{i j}=\frac{\left(D\left(x_{j}, C_{i}\right)\right)^{-1 /(m-1)}}{\sum_{k=1}^{c}\left(D\left(x_{j}, C_{k}\right)\right)^{-1 /(m-1)}}
$$

- Update the cluster centers.

$$
C_{i}=\frac{\sum_{j=1}^{n} U_{i j}^{m} x_{j}}{\sum_{j=1}^{n} U_{i j}^{m}}
$$

- The iterative process starts:

1. Update the $U_{i j}$ by using Eq. (4).

2. Update the $C_{i}$ by using Eq. (5).

3. Update the $D$ using Eq. (3).

4. If $\left|C_{\text {new }}-C_{\text {old }}\right|>\varepsilon ; \quad(\varepsilon=0.001)$ then go to step1

5. Else stop

Assign every pixel to a precise cluster for which the membership value is maximal.

\section{B. Kernel Based FCM}

Kernel version of the FCM algorithm and its objective function are given bellow:

Objective function: $O_{m}(U, C)=\sum_{i=1}^{c} \sum_{j=1}^{n} U_{i j}^{m}\left(1-K\left(x_{j}, C_{i}\right)\right)$

Thus, the revise equations for the essential conditions for minimizing $O_{m}(U, C)$ are given bellow: 


$$
\begin{gathered}
C_{i}=\frac{\sum_{j=1}^{n} U_{i j}^{m} K\left(x_{j}, C_{i}\right) x_{j}}{\sum_{j=1}^{n} U_{i j}^{m} K\left(x_{j}, C_{i}\right)} ; i=1,2, \ldots . C \\
U_{i j}=\frac{\left(1-K\left(x_{j}, C_{i}\right)\right)^{-1 /(m-1)}}{\sum_{k=1}^{c}\left(1-K\left(x_{j}, C_{i}\right)\right)^{-1 /(m-1)}} ; \begin{array}{l}
i=1,2, \ldots . C \\
j=1,2, . ., n
\end{array}
\end{gathered}
$$

We identify the essential conditions for minimizing $O_{m}(U, C)$ are revise Eqs. (6) and (7) only when the kernel function $\mathrm{K}$ is selected to be the Gaussian function with $K\left(x_{j}, C_{i}\right)=\exp \left(-\left\|x_{j}-C_{i}\right\|^{2} / \sigma^{2}\right)$. Different kernels can be selected by replacing the Euclidean distance for different conditions. However, a Gaussian kernel is appropriate for clustering in which it can essentially make the essential conditions. The above proposed KFCM algorithm is very sensitive to the noise conditions. To solve this difficulty Chen and Zhang [16] have introduced the KFCM_S1 and KFCM_S2 techniques which are utilized the spatial data by bring $\alpha$ parameter.

\section{Gaussian Kernal FCM (GKFCM)}

Yang and Tsai [21] have proposed the Gaussian kernel based FCM (GKFCM) for medical image segmentation. The proposed GKFCM algorithm becomes a generalized type of FCM, KFCM_S1 and KFCM_S2 algorithms and presents with more efficiency and robustness. It is mentioned that the parameter $\alpha$ is used to manage the effect of the neighbors for adjusting the spatial bias alteration term. In fact, the parameter $\alpha$ greatly affects the clustering results of KFCM_S1 and KFCM_S2 techniques. Intuitively, it would be appropriate if we can correct each spatial bias alteration term independently for every cluster $i$. That is, the in general parameter $\alpha$ is better replaced with $\dot{\eta}_{i}$ that is correlated to each cluster $i$. In this sense, Yang and Tsai [21] have considered the modified objective function $O_{m}^{G}(U, C)$ with the following constraints.

$$
O_{m}^{G}(U, C)=\sum_{i=1}^{c} \sum_{j=1}^{n} U_{i j}^{m}\left(1-K\left(x_{j}, C_{i}\right)\right)+\sum_{i=1}^{c} \sum_{j=1}^{n} \eta_{i} U_{i j}^{m}\left(1-K\left(\bar{x}_{j}, C_{i}\right)\right)
$$

where $K\left(x_{j}, C_{i}\right)=\exp \left(-\left\|x_{j}-C_{i}\right\|^{2} / \sigma^{2}\right), \bar{x}_{j}$ is the mean of the neighbor pixels, $\sigma^{2}$ is the variance of the total image.

$$
C_{i}=\frac{\sum_{j=1}^{n} U_{i j}^{m}\left(K\left(x_{j}, C_{i}\right) x_{j}+\eta_{i} K\left(\bar{x}_{j}, C_{i}\right) \vec{x}_{j}\right)}{\sum_{j=1}^{n} U_{i j}^{m}\left(K\left(x_{j}, C_{i}\right)+\eta_{i} K\left(\bar{x}_{j}, C_{i}\right)\right)} ; i=1,2, \ldots . C
$$

$U_{i j}=\frac{\left(\left(1-K\left(x_{j}, C_{i}\right)\right)+\eta_{i}\left(1-K\left(\bar{x}_{j}, C_{i}\right)\right)\right)^{-1 /(m-1)}}{\sum_{k=1}^{c}\left(\left(1-K\left(x_{j}, C_{i}\right)\right)+\eta_{i}\left(1-K\left(\bar{x}_{j}, C_{i}\right)\right)\right)^{-1 /(m-1)}} ; \stackrel{i=1,2, \ldots . . C}{j=1,2, . ., n}(10)$

D. Multi-Hyperbolic Tangent and Multi-Gaussian Kernels for FCM (MHMGFCM)

The ideas which are presented in HGTFCM [24] and GKFCM [21] are motivated us to propose the MHMGFCM_S1 and MHMGFCM_S2. The considered hyperbolic tangent function [23] is given bellow.

$$
H\left(x_{j}, C_{i}\right)=1-\tanh \left(-\left\|x_{j}-C_{i}\right\|^{2} / \sigma^{2}\right)
$$

where, $\sigma^{2}$ is the user defined function.

The performance of the segmentation algorithm varies with the $\sigma^{2}$ values. Hence, it is need to fix the appropriate value for $\sigma^{2}$.

In this paper, we consider the value of $\sigma^{2}$ with the variance of the surrounding $P$ neighbors of radius $R$ form the center pixel $x_{j}$.

$\sigma^{2}=\sum_{j=1}^{P}\left\|x_{j}-\bar{x}\right\| / P$

where, $\bar{x}=\sum_{j=1}^{P} x_{j} / P$.

The objective function which is used in this paper for MHMGFCM_S1 and MHMGFCM_S2 is given in Eq. (13).

where, $\bar{x}_{j}$ is the mean for MHMGFCM_S1 and median for MHMGFCM_S2.

\section{MHMGFCM Algorithm}

Input: Raw image; Output: Segmented image;

- Randomly initialize the cluster centers $C_{i}(c=3$ clusters $)$

- Membership values calculation using Eq. (14).

- Cluster centers updating using Eq. (15).

- The iterative process starts:

1. Membership values updating $U_{i j}$ using Eq. (14).

2. Update the cluster centers $C_{i}$ by using Eq. (15).

3. If $\left|C_{\text {new }}-C_{\text {old }}\right|>\xi ;(\xi=0.001)$ then go to step1

4. Else stop

- Assign each pixel to a specific cluster for which the membership value is maximal

$$
\begin{aligned}
& O_{m}^{H}(U, C)=\sum_{i=1}^{c} \sum_{j=1}^{n} U_{i j}^{m}\left(1-H_{1}\left(x_{j}, C_{i}\right)\right)\left(1-H_{2}\left(x_{j}, C_{i}\right)\right)\left(1-K_{1}\left(x_{j}, C_{i}\right)\right)\left(1-K_{2}\left(x_{j}, C_{i}\right)\right) \\
& U_{i j}=\frac{\left(\left(1-H_{1}\left(x_{j}, C_{i}\right)\right)\left(1-H_{2}\left(x_{j}, C_{i}\right)\right)\left(1-K_{1}\left(x_{j}, C_{i}\right)\right)\left(1-K_{2}\left(x_{j}, C_{i}\right)\right)\right)^{-1 /(m-1)}}{\sum_{k=1}^{c}\left(\left(1-H_{1}\left(x_{j}, C_{i}\right)\right)\left(1-H_{2}\left(x_{j}, C_{i}\right)\right)\left(1-K_{1}\left(x_{j}, C_{i}\right)\right)\left(1-K_{2}\left(x_{j}, C_{i}\right)\right)\right)^{-1 /(m-1)}} ; \begin{array}{c}
i=1,2, \ldots . C \\
j=1,2, . ., n
\end{array}
\end{aligned}
$$




$$
C_{i}=\frac{\sum_{j=1}^{n} U_{i j}^{m}\left(\left(K_{1}\left(x_{j}, C_{i}\right) K_{2}\left(x_{j}, C_{i}\right) H_{1}\left(x_{j}, C_{i}\right) H_{2}\left(x_{j}, C_{i}\right)\right) x_{j}\right)}{\sum_{j=1}^{n} U_{i j}^{m}\left(K_{1}\left(x_{j}, C_{i}\right) K_{2}\left(x_{j}, C_{i}\right) H_{1}\left(x_{j}, C_{i}\right) H_{2}\left(x_{j}, C_{i}\right)\right)} ; i=1,2, \ldots . C
$$

\section{EVALUATION MEASURES AND DATASET}

\section{A. Segmentation accuracy/Score}

In this paper, we conside the segmentation accuracy/score as one of the evaluation measures. The score $\left(S_{i k}\right)$ [25], [26] computation between the reference cluster and segmented cluster is defined as follows.

$$
S_{i k}=\frac{A_{i k} \cap A_{r e f k}}{A_{i k} \cup A_{r e f k}}
$$

Where $A_{i k}$ represents the set of pixels belonging to the $k^{\text {th }}$ class computed by the $i^{\text {th }}$ algorithm and $A_{\text {refk }}$ represents the set of pixels belonging to the $k^{\text {th }}$ class in the reference segmented image.

In this paper, we also used the number of iterations (NI) and execution time which are required for the convergence of the algorithm.

TABLE I: MRI DATA ACQUISITION DETAILS [27]
\begin{tabular}{|l|l|}
\hline Sequence & MP-RAGE \\
\hline TR (msec) & $\mathbf{9 . 7}$ \\
\hline TE (msec) & $\mathbf{4 . 0}$ \\
\hline Flip angle (o) & $\mathbf{1 0}$ \\
\hline TI (msec) & $\mathbf{2 0}$ \\
\hline TD (msec) & $\mathbf{2 0 0}$ \\
\hline Orientation & Sagittal \\
\hline Thickness, gap (mm) & $\mathbf{1 . 2 5 , 0}$ \\
\hline Resolution (pixels) & $\mathbf{1 7 6 \times 2 0 8}$ \\
\hline
\end{tabular}

\section{B. MRI Dataset}

The Open Access Series of Imaging Studies (OASIS) [27] is a series of magnetic resonance imaging (MRI) data which is publicly available for study purpose. This data consists of a cross-sectional collected works of 421 subjects aged 18 to 96 years. The MRI acquisition particulars are given in Table I. The performance of the proposed method is tested in terms of score, number of iterations and time. Fig. 1 illustrates the sample images selected for experimentation.

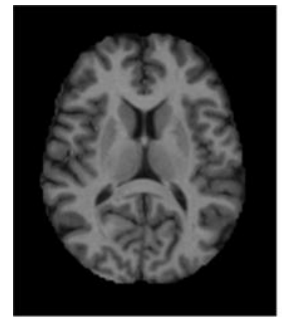

(a)

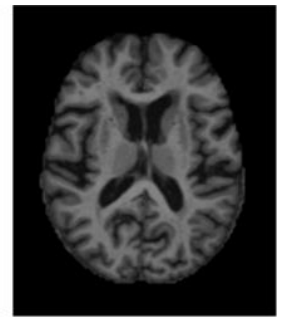

(b)
Reference Image

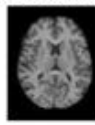

HGFCM_S1

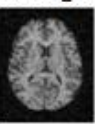

Ref. Class-1
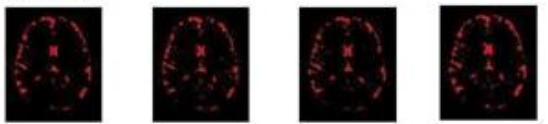

Ref. Class-2
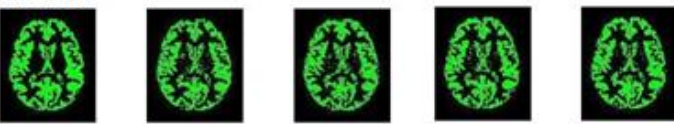

Ref. Class-3
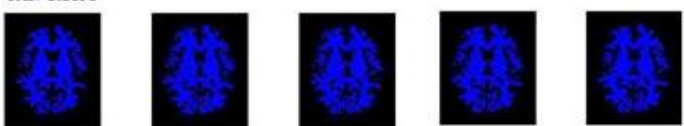

Fig. 2: Comparison of proposed methods (MHMGFCM_S1 and MHMGFCM_S2) with other existing methods (HGFCM_S1 and HGFCM_S2) in terms of three segmented clusters. The original image (Image (a)) are corrupted with 5\% of Gaussian noise.

\section{IV.EXPRIMENTAL RESULTS AND DISCUSSIONS}

In order to verify the effectiveness of the proposed algorithm, experiments were conducted on two brain MRIs [27]. The performance of the proposed algorithm is compared with the other existing FCM variant methods in terms of score, number of iterations (NI) and computational time (CT) on OASIS-MRI dataset.

Fig. 1: Sample images used for experiments 
TABLE II: COMPARISON OF VARIOUS TECHNIQUES IN TERMS OF SCORE ON IMAGE (A) AT DIFFERENT GAUSSIAN NOISES

\begin{tabular}{|c|c|c|c|c|c|c|c|c|c|c|c|c|}
\hline \multicolumn{13}{|c|}{ CL: CLUSTER } \\
\hline \multirow{3}{*}{ Method } & \multicolumn{12}{|c|}{ Gaussian Noise (\%) } \\
\hline & \multicolumn{3}{|c|}{$5 \%$} & \multicolumn{3}{|l|}{$10 \%$} & \multicolumn{3}{|l|}{$15 \%$} & \multicolumn{3}{|l|}{$20 \%$} \\
\hline & Cl-1 & Cl-2 & Cl-3 & Cl-1 & Cl-2 & Cl-3 & Cl-1 & Cl-2 & Cl-3 & Cl-1 & Cl-2 & Cl-3 \\
\hline HGFCM-S1 & 0.68 & 0.77 & 0.84 & 0.59 & 0.70 & 0.81 & 0.52 & 0.69 & 0.81 & 0.49 & 0.62 & 0.75 \\
\hline HGFCM-S2 & 0.68 & 0.78 & 0.86 & 0.54 & 0.70 & 0.81 & 0.53 & 0.65 & 0.77 & 0.46 & 0.62 & 0.76 \\
\hline MHMGFCM_S1 & 0.69 & 0.78 & 0.85 & 0.56 & 0.72 & 0.80 & 0.58 & 0.68 & 0.82 & 0.50 & 0.61 & 0.76 \\
\hline MHMGFCM_S2 & 0.70 & 0.78 & 0.85 & 0.57 & 0.71 & 0.81 & 0.51 & 0.70 & 0.81 & 0.51 & 0.61 & 0.76 \\
\hline
\end{tabular}

TABLE III: COMPARISON OF VARIOUS TECHNIQUES IN TERMS OF NUMBER OF ITERATIONS AND EXECUTION TIME AT DIFFERENT GAUSSIAN NOISE ON IMAGE (B) NI: NUMBER OF ITERATIONS; TM: EXECUTION TIME (SEC.)

\begin{tabular}{lllllllll}
\hline \multirow{2}{*}{ Method } & \multicolumn{9}{l}{ Gaussian Noise } & $\mathbf{1 0 \%}$ & $\mathbf{1 5 \%}$ & $\mathbf{2 0 \%}$ \\
\cline { 2 - 9 } & $\mathbf{5 \%}$ & \multicolumn{10}{c}{ NI } & TM & NI & TM & NI & TM & NI & TM \\
\cline { 2 - 9 } & 21 & 0.38 & 24 & 0.43 & 33 & 0.59 & 24 & 0.43 \\
\hline HGFCM-S1 & 28 & 0.63 & 27 & 0.61 & 24 & 0.54 & 29 & 0.65 \\
\hline HGFCM-S2 & 20 & 0.71 & 20 & 0.45 & 20 & 0.60 & 21 & 0.44 \\
\hline MHMGFCM_S1 & 24 & 0.69 & 19 & 0.62 & 21 & 0.61 & 22 & 0.46 \\
\hline MHMGFCM_S2 & 24
\end{tabular}

Fig. 2 illustrates the cluster segmentation results of the proposed method and other existing methods with the $5 \%$ Gaussian noise on Image (a) of OASIS-MRI dataset. The performance of the proposed methods (MHMGFCM_S1 and MHMGFCM_S2) is compared with the HGFCM_S1 and HGFCM_S2. Table II and Table III show the segmentation performance in terms of score, NI and TM on image (a) and Image (b) respectively under different Gaussian noise conditions. From, Fig. 2 and Tables II to III, it is clear that the proposed method outperforms the other existing algorithms in terms of score, NI and TM.

\section{CONCLUSIONS}

In this paper, new image segmentation algorithms (MHMGFCM_S1 and MHMGFCM_S2) which are increasing the performance and decreasing the computational complexity is proposed. The algorithm utilizes the multi-hyperbolic tangent function and multi-Gaussian kernels. The proposed algorithm is applied on brain MRI which degraded by Gaussian noise. The segmentation results demonstrate that the proposed algorithm shows the robustness under different noises as compared to other existing image segmentation algorithms from FCM family.

\section{REFERENCES}

[1] László Szilágyi, Sándor M. Szilágyi, Balázs Benyó and Zoltán Benyó, "Application of Hybrid c-Means Clustering Models in Inhomogeneity Compensation and MR Brain Image Segmentation," 5th International Symposium on Applied Computational Intelligence and Informatics, pp.105-110, May. 2009.

[2] Orlando J. Tobias, and Rui Seara, "Image Segmentation by Histogram Thresholding Using Fuzzy Sets," IEEE Transactions on Image Processing, vol. 11, no. 12, pp. 1457-1465, 2002.

[3] Arnau Oliver, Xavier Munoz, Joan Batlle, Lluss Pacheco, and Jordi Freixenet, "Improving Clustering Algorithms for Image Segmentation using Contour and Region Information," International Conference on Automation, Quality and Testing, Robotics, Cluj-Napoca, vol. 1, pp. 315-320, 2006.

[4] H. Choi, and R. G. Baraniuk, "Image Segmentation using Waveletdomain Classification," Proc. SPIE Technical Conference on Mathematical Modeling, Bayesian Estimation, and Inverse Problems, Denver, pp. 306-320, 1999.

[5] M. Mary Synthuja Jain Preetha, L. Padma Suresh, and M John Bosco, "Image Segmentation Using Seeded Region Growing," International
Conference on Computing, Electronics and Electrical Technologies, Kumaracoil, pp. 576-583, 2012.

[6] Indira SU and Ramesh A C, "Image Segmentation Using Artificial Neural Network and Genetic Algorithm: A Comparative Analysis," International Conference on Process Automation, Control and Computing (PACC), Coimbatore, pp. 1-6, 2011.

[7] Dipti Patra and P. K. Nanda, "Image Segmentation Using Markov Random Field Model Learning Feature and Parallel Hybrid Algorithm," International Conference on Computational Intelligence and Multimedia Applications, Sivakasi, Tamil Nadu, pp. 400-407, 2007.

[8] MacQueen,J.B. "Some Methods for classification and Analysis of Multivariate Observations,"Proceedings of 5th Berkeley Symposium on Mathematical Statistics and Probability. University of California Press, pp. 281-297, 1967

[9] L. Zadeh, "Fuzzy sets," Inf. Control, vol. 8, pp. 338-353, 1965.

[10] J. Udupa and S. Samarasekera, "Fuzzy connectedness and object definition: Theory, algorithm and applications in image segmentation," Graphical Models and Image Processing, vol. 58, no. 3, pp. 246-261, 1996.

[11] J. C. Noordam and W. H. A. M. van den Broek, "Multivariate image segmentation based on geometrically guided fuzzy C-means clustering," Journal of Chemometrics, vol. 16, 1 - 11, 2002.

[12] Y. Tolias and S. Panas, "Image segmentation by a fuzzy clustering algorithm using adaptive spatially constrained membership functions," IEEE Transactions on Systems, Man, and Cybernetics, vol. 28, no. 3 pp. 359-369, Mar.1998.

[13] J.C. Noordam, W.H.A.M. van den Broek, and L.M.C. Buydens, " Geometrically Guided Fuzzy C-means Clustering for Multivariate Image Segmentation," Proc. Int. Conf. on Pattern Recognition, Barcelona, vol. 1, 462 - 465, 2000.

[14] D. Pham, "Fuzzy clustering with spatial constraints," in Proceedings of International Conference on Image Processing, New York, 2002, vol. II, pp. 65-68.

[15] M. Ahmed, S. Yamany, N. Mohamed, A. Farag, and T. Moriarty, "A modified fuzzy C-means algorithm for bias field estimation and segmentation of MRI data," IEEE Transactions on Medical Imaging, vol. 21, no. 3, pp. 193-199, 2002.

[16] S. Chen and D. Zhang, "Robust image segmentation using FCM with spatial constraints based on new kernel-induced distance measure," IEEE Transactions on Systems, Man, and Cybernetics, vol. 34, pp. 1907-1916, 2004.

[17] L. Szilagyi, Z. Benyo, S. Szilagyii, and H. Adam, "MR brain image segmentation using an enhanced fuzzy C-means algorithm," in Proceedings of the 25" Annual International Conference of the IEEE EMBS, pp. 17-21, 2003. 
[18] M. Krinidis and I. Pitas, "Color texture segmentation based-on the modal energy of deformable surfaces," IEEE Transactions on Image Processing, vol. 18, no. 7, pp. 1613-1622, Jul. 2009.

[19] M. Yang, Y. J. Hu, K. Lin, and C. C. Lin, "Segmentation techniques for tissue differentiation in MRI of ophthalmology using fuzzy clustering algorithms," Magnetic Resonance Imaging, vol. 20, no. 2, pp. 173-179, 2002.

[20] W. Cai, S. Chen, and D. Zhang, "Fast and robust fuzzy c-means clustering algorithms incorporating local information for image segmentation," Pattern Recognition, vol. 40, no. 3, pp. 825-838, Mar. 2007.

[21] Miin-Shen Yang, Hsu-Shen Tsai, "A Gaussian kernel-based fuzzy cmeans algorithm with a spatial bias correction," Pattern Recognition Letters, vol. 29, pp. 1713-1725, May 2008.

[22] Long Chen, C. L. Philip Chen, and Mingzhu Lu, "A Multiple-Kernel Fuzzy C-Means Algorithm for Image Segmentation," IEEE Trans. Systems, Man, And Cybernetics-Part B: Cybernetics, vol. 41, No. 5, pp. 1263 - 1274, February 9, 2011.

[23] S.R. Kannan, S. Ramathilagam, R. Devi, A. Sathya, " Robust kernel FCM in segmentation of breast medical images," Expert Systems with Applications, vol. 38, 4382-4389, 2011.

[24] Venu N and Anuradha B, " Integration of Hyperbolic Tangent and Gaussian Kernels for Fuzzy C-means Algorithm with Spatial Information for MRI Segmentation," Fifth International Conference on Advanced Computing (ICoAC 2013), Anna University, Chennai, India, 2013.

[25] Masulli, F., Schenone, A., "A fuzzy clustering based segmentation system as support to diagnosis in medical imaging," Artif. Intell. Med. vol. 16, pp. 129-147, 1999.

[26] Zhang, D.Q., Chen, S.C., "A novel kernelized fuzzy c-means algorithm with application in medical image segmentation," Artif. Intell. Med., vol. 32, pp. 37-50, 2004.

[27] D. S.Marcus, T. H. Wang, J. Parker, J. G. Csernansky, J. C.Morris, and R. L. Buckner, Open access series of imaging studies (OASIS): Crosssectional MRI data in young, middle aged, nondemented, and demented older adults. J. Cogn. Neurosci., 19 (9) 1498-1507, 2007. 